\title{
Discrete Weighted Least-Squares Method for the Poisson and Biharmonic Problems on Domains with Smooth Boundary
}

\author{
P. Žitñan \\ Department of Mathematical Analysis and Applied Mathematics \\ Faculty of Science, University of Žilina, Hurbanova 15, 01026 Žilina, Slovakia \\ peter.zitnan@fpv.uniza.sk
}

In this article a discrete weighted least-squares method for the numerical solution of elliptic partial differential equations exhibiting smooth solution is presented. It is shown how to create well-conditioned matrices of the resulting system of linear equations using algebraic polynomials, carefully selected matching points and weight factors. Two simple algorithms generating suitable matching points, the Chebyshev matching points for standard two-dimensional domains and the approximate Fekete points of Sommariva and Vianello for general domains, are described. The efficiency of the presented method is demonstrated by solving the Poisson and biharmonic problems with the homogeneous Dirichlet boundary conditions defined on circular and annular domains using basis functions satisfying and not satisfying the prescribed boundary conditions.

Keywords: discrete least-squares method, Fekete points, matrix conditioning, Poisson and biharmonic problems, circular and annular domains

\section{INTRODUCTION}

The least-squares method with a variety of its modifications is a generally accepted tool for the numerical solution of many important problems in engineering and applied mathematics. Particularly, it is well suited for the numerical solution of problems governed by partial differential equations. Although the articles concerning the numerical solution of partial differential equations using its pure discrete form are very rare in the literature $[1,2]$, the continuous form in connection with the finite element approximation is a very attractive method for the solution of many boundary value problems using direct and mixed formulations [3]. This acceptance is evident in spite of two nontrivial bottlenecks of the least-squares approach. Firstly, because of the residual error minimization principle behind the least-squares approach, this method may be used only for boundary value problems exhibiting sufficiently smooth solution with the square-integrable highest derivatives. Consequently, in contrast to the Ritz method, it is not possible to use this method for problems exhibiting weak solution. Important practical consequence of the residual minimization principle is that the convergence of the least-squares approximation in solving Poisson and biharmonic problems depends on the convergence of its second and fourth derivatives, respectively. This is an essential disadvantage in comparison with the Ritz method, where only the convergence of the first derivatives of the Ritz approximation is required when solving the Poisson problems and the convergence of the second derivatives is required when solving the biharmonic problems. Secondly, the resulting coefficient matrices are essentially more ill-conditioned than the 
Ritz matrices.

On the other hand, the discrete least-squares method exhibits very useful properties. The coefficient matrix of the resulting system of linear equations is symmetric and positive definite. Although the method is variational, it does not require numerical integration and may, in principle, be used for solving boundary value problems defined on domains with a complicated boundary shape. Such problems may be solved using trial functions not satisfying the prescribed boundary conditions, whereas these conditions may again be imposed by a discrete least-squares technique.

In this paper we will consider the efficient choice of matching points and weight factors for the discrete weighted least-squares method applied to the Poisson and biharmonic problems defined on circular and annular domains. By virtue of extensive numerical experiments we will identify simply computable sets of matching points for the above mentioned domains giving well-conditioned matrices and high precision results.

\section{DISCRETE LEAST-SQUARES METHOD}

Let us consider a differential equation of the form

$$
\mathcal{A} u(\mathbf{x})=f(\mathbf{x}), \quad \mathbf{x} \in \Omega,
$$

subject to the homogeneous Dirichlet boundary conditions

$$
\mathcal{B} u(\mathbf{x})=0, \quad \mathbf{x} \in \partial \Omega,
$$

where $\Omega$ is an open two-dimensional bounded domain with the Lipschitz boundary $\partial \Omega$. According to the classical regularity results for boundary value problems of the form (1)-(2), if $f \in L_{2}(\Omega)$, the corresponding solution belongs to the Sobolev spaces $W_{2}^{1}(\Omega)$ and $W_{2}^{2}(\Omega)$ for $\mathcal{A}=-\Delta$ and $\mathcal{A}=\Delta^{2}$, respectively. The solution of higher regularity may be obtained by enforcing specific requirements on smoothness of the boundary $\partial \Omega$, boundary conditions and the right-hand side function $f$ [4]. For the sake of simplicity we will consider only the problems with an infinitely differentiable solution for all $\mathbf{x} \in \Omega \cup \partial \Omega$. This reduction is suitable also from the practical point of view, because we will try to obtain accurate approximations using the algebraic polynomials. The presence of boundary or interior singularities requires special treatment and such problems are not suitable for polynomial solution.

The main idea behind the discrete weighted least-squares method (DWLSM) is to find an approximate solution minimizing a residual error of the problem (1)-(2). If we assume the approximate solution $u_{n}(\mathbf{x})$ in the form $u_{n}(\mathbf{x})=\sum_{j=1}^{n} c_{j} \varphi_{j}(\mathbf{x})$, where the basis functions $\varphi_{j}(\mathbf{x})$ satisfy the prescribed boundary conditions, the discrete weighted least-squares method results in the minimization of the weighted residual error function

$$
E_{m, n}(\mathbf{c})=\sum_{k=1}^{m} w_{k}\left(\sum_{j=1}^{n} c_{j} \mathcal{A} \varphi_{j}\left(\mathbf{p}_{k}\right)-f\left(\mathbf{p}_{k}\right)\right)^{2}
$$

where $\mathbf{c}=\left(c_{1}, c_{2}, \ldots, c_{n}\right), \mathbf{p}_{k}=\left(x_{k}, y_{k}\right)$ are matching points inside $\Omega$ and $w_{k}>0$ are weight factors which will be specified later. The necessary conditions for $E_{m, n}(\mathbf{c})$ 
to have a minimum

$$
\frac{\partial E_{m, n}(\mathbf{c})}{\partial c_{i}}=0, \quad i=1,2, \ldots, n
$$

give the system of linear equations

$$
A c=b,
$$

where

$$
a_{i, j}=\sum_{k=1}^{m} w_{k} \mathcal{A} \varphi_{i}\left(\mathbf{p}_{k}\right) \mathcal{A} \varphi_{j}\left(\mathbf{p}_{k}\right), \quad b_{i}=\sum_{k=1}^{m} w_{k} \mathcal{A} \varphi_{i}\left(\mathbf{p}_{k}\right) f\left(\mathbf{p}_{k}\right) .
$$

There is also the possibility to solve (1)-(2) by a least-squares technique resulting in the solution of an over-determined system of linear equations [5], however, we will prefer the presented approach because of its simple use for the solution of eigenvalue problems [6].

\section{NUMERICAL RESULTS}

The aim of this section is to present extensive numerical experiments with DWLSM used for computing the approximate solution of the Poisson and biharmonic problems defined on the standard two-dimensional domains - disc and concentric annulus. In these experiments algebraic polynomials orthogonal on the unit disc and different sets of matching points have been used in order to show how to use DWLSM in an efficient form. Because the optimal choice of the matching points for DWLSM is not considered in the literature, we will help with some results from the theory of function interpolation. Denoting $I_{n} f$ the interpolating polynomial from the space $P_{n}$ of algebraic polynomials of order $n$ or less, i.e., $I_{n} f\left(\mathbf{x}_{k}\right)=f\left(\mathbf{x}_{k}\right)$, for $k=0,1,2, \ldots, n$, where $\mathbf{x}_{k}$ are the interpolation nodes, the known Lebesgue lemma shows how well $f$ can be approximated by its interpolating polynomial $I_{n} f$ in the maximum norm

$$
\left\|f-I_{n} f\right\|_{\infty} \leq\left(1+\Lambda_{n}\right)\|f-g\|_{\infty} .
$$

In this inequality $g \in P_{n}$ stands for the best polynomial approximation of $f$ and $\Lambda_{n}$ is known as the Lebesgue constant.

The optimal interpolating nodes which minimize the Lebesgue constant are called the Lebesgue points. As written in [7]: "Almost nothing seems to be known about Lebesgue points in more than one dimension. Nor are we aware of a feasible method for computing them numerically." In spite of this conclusion, there are sets of points giving near-optimal values of the Lebesgue constant for the square domain [8-10]. On the other hand, as stated in the introduction, the crucial limitation of the least-squares method we would have in mind is ill-conditioning of the coefficient matrix $A$. It means that instead of searching for the Lebesgue points for a domain $\Omega$, we have to search for matching points ensuring a compromise between two requirements. Firstly, we want to compute the most accurate approximation of (1)-(2). Secondly, to achieve the highest possible accuracy we need the coefficient matrix $A$ with condition number as small as possible. Clearly, this two-fold optimization problem is far from being an easy task with respect to both a theoretical and practical point of view. Fortunately, as seen from our computational experiments, there are other sets of matching points resulting in a very accurate solution of (1)-(2), and well conditioned matrices as well. 
Such points frequently used in engineering practice are the Fekete points. To determine the Fekete points for a domain $\Omega$ we need to select a basis $\varphi_{j}$ for $P_{n}$. The Fekete points for a domain $\Omega$ are those points $\mathbf{x}_{i} \in \Omega$ which maximize the determinant of the Vandermonde matrix $V$ with the elements $v_{i j}=\varphi_{j}\left(\mathbf{x}_{i}\right)$. As seem from the extensive author search in the literature, there are only two planar regions, square and triangle, for which the Fekete points are known. In the square case these points are determined by the Cartesian product of the one-dimensional Gauss-Lobatto points [11], while in the triangle case the Fekete points and different sets of extremal points are computed [7, 12 - 16]. A common feature of all these sets of points is that the points are concentrated near the boundary of the region under consideration and the concentration of the points is proportional to the concentration of the zeros of orthogonal polynomials. These properties we will have in mind in the construction of suitable matching points for the unit disc and concentric annulus.

To improve the condition number of the coefficient matrix $A$ for each set of selected matching points the weight factors $w_{k}$ may be used as well. Similarly, as in the Gauss integration formulae, where the integration points near the boundary of the region of integration have smaller weights than the interior points, the weights $\mathbf{w}=\left(w_{1}, w_{2}, \ldots, w_{m}\right)$ corresponding to the set of matching points $\left\{\mathbf{p}_{k}\right\}_{k=1}^{m}$ have been determined by the one-dimensional minimization

$$
\mathbf{w}=\arg \min _{\alpha \in\langle 0, a\rangle} \operatorname{cond}\left(A_{\alpha}\right),
$$

where $\operatorname{cond}\left(A_{\alpha}\right)$ denotes the spectral condition number of the coefficient matrix $A_{\alpha}$ parametrically dependent on $\alpha, A_{\alpha} \equiv A\left(\mathbf{w}_{\alpha}\right)$ for $\mathbf{w}_{\alpha}=\left(w_{1}(\alpha), w_{2}(\alpha), \ldots, w_{m}(\alpha)\right)$ and

$$
w_{k}(\alpha)=W_{\alpha}\left(x_{k}, y_{k}\right), \quad k=1,2, \ldots, m .
$$

The weight function $W_{\alpha}(x, y)$ may be considered a positive function on the region $\Omega$ decreasing for points which tend to the boundary $\partial \Omega$.

\section{POISSON PROBLEM ON THE UNIT DISC}

To solve the Poisson problem (1)-(2) defined on the unit disc $\Omega$ subject to the homogeneous Dirichlet boundary condition $u=0$ on $\partial \Omega$ the basis functions [17]

$$
\varphi_{k, l}^{\tau}(x, y)=\left(1-x^{2}-y^{2}\right) J_{l}^{\tau}\left(x \cos \frac{k \pi}{l+1}+y \sin \frac{k \pi}{l+1}\right), \quad 0 \leq k \leq l,
$$

have been used, where $J_{l}^{\tau}(x)$ are the Jacobi polynomials orthogonal on $\langle-1,1\rangle$ with the weight function $\left(1-x^{2}\right)^{\tau}$.

Between two possibilities of how to construct suitable disc matching points to transform suitable matching points for the square into the unit disc or to construct an algorithm creating suitable matching points directly, we have decided for a simple construction generating disc matching points in an explicit form. This construction generates different sets of disc points, where each set of points is determined by three parameters $m_{1}, m_{2}, m_{3}$. The Chebyshev disc points are situated on $m_{3}$ circles with the radii $r_{i}=z_{i}^{+}, i=1,2, \ldots, m_{3}$, where $z_{i}^{+}$are positive roots of the Chebyshev polynomial of order $2 m_{3}$. The first parameter $m_{1}$ denotes the number of points situated on the smallest circle. The second parameter $m_{2}$ denotes the increment of points between each pair of the neighboring circles. The points 
situated on each even circle are rotated on half the distance between two adjacent points of these circles. Among the sets of Chebyshev disc points generated for different values of $m_{1}, m_{2}$, and $m_{3}$ the sets that have been taken for computation are those having minimal number of points and giving almost minimal condition number of the corresponding coefficient matrices $A_{\alpha}$, i.e., using more points results in a negligible improvement of matrix conditioning. Such easily computable matching points giving small condition number of the coefficient matrices using minimal number of points we will consider as points feasible for computation and denote in short as FCD-points (feasible Chebyshev disc points). Fig.1 shows 2080 FCD-points determined by the parameters $m_{1}=3, m_{2}=4, m_{3}=32$.

The spectral condition numbers of the coefficient matrices $A_{\alpha_{o p t}}$ corresponding to FCD-points using $n=100,200,400,800,1600$ basis functions (4) for $\tau=0$ are shown in the logarithmic form in Table 1 . In these computations the weight function

$$
W_{\alpha}(x, y)=\left(1-x^{2}-y^{2}\right)^{\alpha}
$$

has been used for optimization of the weight factors $w_{k}$.

Finally, it remains to show that the considered sets of matching points, besides small condition numbers of the corresponding coefficient matrices $A_{\alpha_{\text {opt }}}$, produce high precision results as well. For the sake of simplicity the coefficient $\alpha_{\text {opt }}$ in Table 1 and Table 4 is denoted as $\alpha$. In Table 2 an error of the approximate solution in the form

$$
\log _{10}\left(\max _{1 \leq i, j \leq 50} \frac{\left|u_{\text {approx }}\left(x_{i}, y_{j}\right)-u_{\text {exact }}\left(x_{i}, y_{j}\right)\right|}{c}\right)
$$

is reported for the exact solution

$$
u_{\text {exact }}(x, y)=\left(1-x^{2}-y^{2}\right) \sin (\gamma x y)
$$

where $\gamma$ is a shape diversity parameter. In this table the sets of FCD-points reported in Table 1 have been used for three values of the parameter $\gamma$. For $\gamma=1,20$ and 30 the exact solution (7) exhibits two positive maxima separated by two negative minima, six positive maxima separated by six negative minima and ten positive maxima separated by ten negative minima, respectively. In $(6)\left(x_{i}, y_{j}\right)$ are points of the equidistant mesh of $\Omega$ for which $x_{i+1}-x_{i}=y_{j+1}-y_{j}=1 / 51$ and $c=$ $\max _{\mathbf{x} \in \Omega}\left|u_{\text {exact }}(\mathbf{x})\right|$.

\section{BIHARMONIC PROBLEM ON THE UNIT DISC}

The biharmonic problem (1)-(2) defined in the unit disc $\Omega$ subject to the homogeneous Dirichlet boundary conditions $u=\partial u / \partial n=0$ on $\partial \Omega$, where $n$ is the outward normal direction to $\partial \Omega$, has been solved in the case when exact solution is of the form

$$
u_{\text {exact }}(x, y)=\left(1-x^{2}-y^{2}\right)^{2} \sin (\gamma x y) .
$$

In the numerical computation presented in Table 1 and Table 3 the basis functions

$$
\varphi_{k, l}^{\tau}(x, y)=\left(1-x^{2}-y^{2}\right)^{2} J_{l}^{\tau}\left(x \cos \frac{k \pi}{l+1}+y \sin \frac{k \pi}{l+1}\right), \quad 0 \leq k \leq l,
$$

for $\tau=0$ and FCD-points have been used. The corresponding condition numbers of the coefficient matrices $A_{\alpha}$ are shown in Table 1 . These values have been computed for the same sets of FCD-points and the weight function (5) as for the Poisson 
problem. Among the sets of the Chebyshev disc points used in our numerical experiments the ones generated by the parameters $\left(3,4, m_{3}\right)$ produce the most suitable points.

\section{POISSON PROBLEM ON THE CONCENTRIC ANNULUS}

For the solution of the Poisson problem (1)-(2) defined on the unit concentric annulus with the inner circle radius $R_{1}=0.2$ the basis functions

$$
\varphi_{k, l}^{\tau}(x, y)=\left(1-x^{2}-y^{2}\right)\left(R_{1}^{2}-x^{2}-y^{2}\right) J_{l}^{\tau}\left(x \cos \frac{k \pi}{l+1}+y \sin \frac{k \pi}{l+1}\right), \quad 0 \leq k \leq l,
$$

have been used for $\tau=0$ together with matching points created by a procedure similar to the one proposed for the Chebyshev disc points. The corresponding Chebyshev annulus matching points are determined by three parameters $m_{1}, m_{2}, m_{3}$, where $m_{1}$ denotes the number of points situated on the smallest circle, $m_{2}$ denotes the increment of points between each pair of the neighboring circles, and $m_{3}$ denotes the number of circles inside the annulus containing the desired matching points. The radii of these circles are the values of the zeros of the Chebyshev polynomial of order $m_{3}$ transformed into the interval $\left\langle R_{1}, 1\right\rangle$. The points situated on each even circle are rotated on half the distance between two adjacent points of these circles.

The computations presented in Table 4 show the parameters $m_{1}, m_{2}, m_{3}$ creating the sets of FCA-points (feasible Chebyshev annulus points), the values of the optimization parameter $\alpha$ giving the optimal weight factors $w_{k}$ with respect to the weight function

$$
W_{\alpha}(x, y)=\left(\left(1-x^{2}-y^{2}\right)\left(R_{1}^{2}-x^{2}-y^{2}\right)\right)^{\alpha}
$$

and the values $\log _{10}\left(\operatorname{cond}\left(A_{\alpha}\right)\right)$ for the corresponding coefficient matrices $A_{\alpha}$ using $n=100,200,400,800,1600$ basis functions (9). These sets of FCA-points have been used for computing the approximate solution of the Poisson problem (1)-(2) subject to the homogeneous Dirichlet boundary condition with the exact solution

$$
u_{\text {exact }}(x, y)=\left(1-x^{2}-y^{2}\right)\left(R_{1}^{2}-x^{2}-y^{2}\right) \sin (\gamma x y) .
$$

The error bounds of the form (6) obtained for $\gamma=1,20,30$ are shown in Table 5. Fig. 2 shows $2261 \mathrm{FCA}$-points determined by the parameters $m_{1}=17, m_{2}=3, m_{3}=17$.

\section{BIHARMONIC PROBLEM ON THE CONCENTRIC ANNULUS}

The next example to be considered in this article certifies applicability of the presented DWLSM for solving the first biharmonic problem defined on a doubleconnected domain. The biharmonic problem defined on the unit concentric annulus with the inner circle radius $R_{1}=0.2$ has been solved for the exact solution

$$
u_{\text {exact }}(x, y)=\left(1-x^{2}-y^{2}\right)^{2}\left(R_{1}^{2}-x^{2}-y^{2}\right)^{2} \sin (\gamma x y)
$$

satisfying the homogeneous Dirichlet boundary conditions $u=\partial u / \partial n=0$ on $\partial \Omega$. In the solution of this problem the basis functions

$$
\varphi_{k, l}^{\tau}(x, y)=\left(1-x^{2}-y^{2}\right)^{2}\left(R_{1}^{2}-x^{2}-y^{2}\right)^{2} J_{l}^{\tau}\left(x \cos \frac{k \pi}{l+1}+y \sin \frac{k \pi}{l+1}\right), 0 \leq k \leq l,
$$

and the same sets of FCA-points as in the Poisson problem have been used. The corresponding condition numbers $\left(\log _{10}\left(\operatorname{cond}\left(A_{\alpha}\right)\right)\right)$ optimized with respect to the 
weight function (10) using $n=100,200,400,800,1600$ basis functions (11) with $\tau=0$ are shown in Table 4 . The resulting error bounds of the form (6) for different values of the shape diversity parameter $\gamma$ are reported in Table 6 .

\section{PROBLEMS ON GENERAL DOMAINS}

The Poisson and biharmonic problems solved above are considered only for standard domains and the basis functions used in the presented computations satisfy the prescribed boundary conditions. However, in practice it is of interest to solve problems defined on domains with a complicated boundary shape and domains with holes using basis functions not satisfying any of the prescribed boundary conditions. To show the ability of the presented DWLSM to solve the problems on general domains with a smooth boundary we will consider the Poisson and biharmonic problems on the unit disc with the homogeneous Dirichlet boundary conditions using basis functions not satisfying these boundary conditions. The matching points used in solving these problems will be generated by a recently developed method of Sommariva and Vianello (Computing approximate Fekete points by QR factorizations of Vandermonde matrices, submitted for publication).

To create $m$ matching points inside a general domain $\Omega$ using the method of Sommariva and Vianello we need to choose an initial set of points $P_{M}=\left\{\mathbf{x}_{i} \in\right.$ $\Omega, 1 \leq i \leq M, M>m\}$ and to form the corresponding Vandermonde matrix $V_{M}\left(v_{i j}=\varphi_{j}\left(\mathbf{x}_{i}\right), 1 \leq j \leq m, \mathbf{x}_{i} \in P_{M}, 1 \leq i \leq M\right)$, where $\varphi_{j}(\mathbf{x})$ are the used basis functions. Denoting $\mathbf{b}$ a nonzero $m$-dimensional vector, the solution of the undetermined system of linear equations

$$
V_{M}^{T} \mathbf{z}=\mathbf{b}
$$

by the QR decomposition with column pivoting returns a vector $\mathbf{z}$. The all nonzero entries $z_{k}$ of $\mathbf{z}$ identify the corresponding points $\mathbf{x}_{k}$ maximizing the determinant of the corresponding $m \times m$ Vandermonde submatrix $V_{m}$ over all $m$-points subsets of $P_{M}$. Figure 3 shows 1800 approximate Fekete points selected from 3150 Chebyshev disc points. For more details on how to compute the approximate Fekete points the mentioned article of Sommariva and Vianello (www.math.unipd.it/ marcov/pdf/afek.pdf) should be consulted.

Using basis functions not satisfying the prescribed boundary conditions, a small modification of the residual error function have to be made. The modified error function is of the form

$$
E_{m, n}(\mathbf{c})=\sum_{k=1}^{m} w_{k}\left(\sum_{j=1}^{n} c_{j} \Delta \varphi_{j}\left(\mathbf{p}_{k}\right)+f\left(\mathbf{p}_{k}\right)\right)^{2}+c_{1} \sum_{k=1}^{n b p}\left(\sum_{j=1}^{n} c_{j} \varphi_{j}\left(\mathbf{p}_{k}^{b}\right)\right)^{2}
$$

in the Poissosn problem, and

$$
\begin{gathered}
E_{m, n}(\mathbf{c})=\sum_{k=1}^{m} w_{k}\left(\sum_{j=1}^{n} c_{j} \Delta^{2} \varphi_{j}\left(\mathbf{p}_{k}\right)-f\left(\mathbf{p}_{k}\right)\right)^{2}+c_{1} \sum_{k=1}^{n b p}\left(\sum_{j=1}^{n} c_{j} \varphi_{j}\left(\mathbf{p}_{k}^{b}\right)\right)^{2} \\
+c_{2} \sum_{k=1}^{n b p}\left(\sum_{j=1}^{n} c_{j} \frac{\partial \varphi_{j}}{\partial n}\left(\mathbf{p}_{k}^{b}\right)\right)^{2}
\end{gathered}
$$


in the biharmonic problem, where $c_{1}$, and $c_{2}$ denote the weight factors ensuring a balance between the error required for the interior matching points and the error required for the boundary matching points. The boundary matching points $\mathbf{p}_{k}^{b}, 1 \leq$ $k \leq n b p$, are located equidistantly on the unit circle. Consequently, the entries of the corresponding coefficient matrices have to be modified as follows

$$
a_{i, j}=\sum_{k=1}^{m} w_{k} \Delta \varphi_{i}\left(\mathbf{p}_{k}\right) \Delta \varphi_{j}\left(\mathbf{p}_{k}\right)+c_{1} \sum_{k=1}^{n b p} \varphi_{i}\left(\mathbf{p}_{k}^{b}\right) \varphi_{j}\left(\mathbf{p}_{k}^{b}\right)
$$

in the Poisson problem, and

$$
a_{i, j}=\sum_{k=1}^{m} w_{k} \Delta^{2} \varphi_{i}\left(\mathbf{p}_{k}\right) \Delta^{2} \varphi_{j}\left(\mathbf{p}_{k}\right)+c_{1} \sum_{k=1}^{n b p} \varphi_{i}\left(\mathbf{p}_{k}^{b}\right) \varphi_{j}\left(\mathbf{p}_{k}^{b}\right)+c_{2} \sum_{k=1}^{n b p} \frac{\partial \varphi_{i}}{\partial n}\left(\mathbf{p}_{k}^{b}\right) \frac{\partial \varphi_{j}}{\partial n}\left(\mathbf{p}_{k}^{b}\right)
$$

in the biharmonic problem.

To avoid the zero rows and columns of the coefficient matrices, the basis functions

$$
\varphi_{k, l}^{\tau}(x, y)=\left(R^{2}-x^{2}-y^{2}\right)^{E x p} J_{l}^{\tau}\left(x \cos \frac{k \pi}{l+1}+y \sin \frac{k \pi}{l+1}\right), \quad 0 \leq k \leq l,
$$

have been used, where $\operatorname{Exp}=1$ in the Poisson problem, and $\operatorname{Exp}=2$ in the biharmonic problem. The inequality $R>1$ ensures that the basis functions (12) do not satisfy the prescribed homogeneous Dirichlet boundary conditions on the unit circle. In the presented numerical experiments the initial sets of points $P_{M}$ are filled up by the Chebyshev disc points which give better approximate Fekete points than the Cartesian product of the one-dimensional equidistant points. The resulting values of the spectral condition numbers for the Poisson problem defined on the unit disc are shown in Table 7 for the non-optimized $(\alpha=0)$ and optimized $(\alpha=1.8)$ coefficient matrices $A_{\alpha}$. In the reported computations $c_{1} \in\left(10^{2}, 10^{3}\right), \tau=0$, and $R=5$. The corresponding error bounds (6) for different values of the shape diversity parameter $\gamma$ are listed in Table 8. Similarly, the resulting values of the spectral condition numbers for the biharmonic problem defined on the unit disc are shown in Table 9 for the non-optimized $(\alpha=0)$ and optimized $(\alpha=3.8)$ coefficient matrices $A_{\alpha}$. In the reported computations $c_{1}, c_{2} \in\left(10^{4}, 10^{6}\right), \tau=0$ and $R=5$. The corresponding error bounds (6) for different values of the shape diversity parameter $\gamma$ are listed in Table 10 .

\section{CONCLUSIONS}

In this article it is shown how to create well-conditioned coefficient matrices in the numerical solution of the Poisson and biharmonic problems defined on twodimensional domains with a smooth boundary using DWLSM . Moreover, it is the author belief that the presented approach may be generalized for any second and fourth order boundary value problem exhibiting infinitely differentiable solution. The success of the solution requires the basis functions composed by algebraic polynomials orthogonal or at least near-orthogonal on the considered domain and carefully selected matching points. Although the crucial point of the presented approach - the construction of suitable matching points for DWLSM is not considered in the literature, there are three different sets of points which may successfully be used in the solution of the above mentioned problems. 
The explicit construction of the Chebyshev matching points may be a good choice at least for simply connected and double-connected domains with the boundaries parametrized in polar coordinates. On the other hand, the method of Sommariva and Vianello, giving the approximate Fekete points, and the method of Bendito and coworkers [18], giving the exact Fekete points, offer very promising alternatives for any domain with Lipschitz boundary. Mainly the method of Sommariva and Vianello, owing to its simplicity and efficiency, deserves attention of mathematicians and engineers. The sets of matching points presented in this article enable high precision computation of the resulting systems of linear equations up to the order 1600. Although the condition numbers of these matrices are large, the round-off error propagates relatively slowly. For example, in the biharmonic problem on the annulus, where $\log _{10}\left(\operatorname{cond}\left(A_{\alpha}\right)\right)=11.0$, the approximate solution has been computed with only the last 4 digits destroyed by the round-off error (see Table 4 and Table 6). This case implies that the resulting accuracy may be better than predicted according to the actual condition number of the coefficient matrices.

Moreover, conditioning of the coefficient matrices corresponding to an arbitrary set of matching points may be improved by the optimization of the weight factors $w_{k}$. In the presented computations, using the basis functions not satisfying the prescribed boundary conditions (Table 7 and Table 9), the simple onedimensional optimization (3) is able to improve matrix conditioning significantly from $\log _{10}\left(\operatorname{cond}\left(A_{0}\right)\right)=8.5$ to $\log _{10}\left(\operatorname{cond}\left(A_{1.8}\right)\right)=4.5$ in the Poisson problem and from $\log _{10}\left(\operatorname{cond}\left(A_{0}\right)\right)=17.6$ to $\log _{10}\left(\operatorname{cond}\left(A_{3.8}\right)\right)=9.2$ in the biharmonic problem. The interesting question remains how to formulate a simple optimization criterion giving better improvement of matrix conditioning. In principle, the optimal weight factors with respect to conditioning of the coefficient matrices have to be computed by solving the minimization problem

$$
\mathbf{w}_{\text {opt }}=\arg \min _{\mathbf{w} \in R_{m}^{+}} \operatorname{cond}(A(\mathbf{w})) .
$$

Similarly, conditioning of the coefficient matrices may be optimized directly searching for the conditionally optimal matching points $\left\{\mathbf{p}_{i, o p t}\right\}_{i=1}^{m}$ defined as

$$
\left\{\mathbf{p}_{i, o p t}\right\}_{i=1}^{m}=\arg \min _{\mathbf{p}_{i} \in \Omega, i=1,2, \ldots, m} \operatorname{cond}\left(A\left(\mathbf{p}_{i}\right)\right) .
$$

However, booth these problems are difficult nonlinear optimization problems and their efficient solution will be the scope of our future research.

As shown in our numerical experiments in order to obtain the coefficient matrices with the condition number as small as possible, the basis functions orthogonal or at least near-orthogonal on the considered domain are required as well. Because the near-orthogonality and particularly the orthogonality of the basis functions may cause serious difficulties in solving the problems defined on highly non-convex and multiple connected domains, different types of the used approximation employing radial basis functions [19] and splines [20] may be considered. Consequently, the question how to construct suitable basis functions for the discrete least-squares method applied to boundary value problems on irregular domains could open another space for our future research.

Acknowledgement: The author wishes to thank one of the referees for helpful comments improving the presentation of this article. 
This article was partially supported by Grant VEGA 2/0097/08.

\section{References}

[1] J. P. Laible and G. F. Pinder, Least squares collocation solution of differential equations on irregularly shaped domains using orthogonal meshes, Numer Method Partial Differential Equations 5 (1989), 347.

[2] U. L. Pen, Discrete least-squares global approximations to solutions of partial differential equations, Numer Method Partial Differential Equations 7 (1991), 303.

[3] P. B. Bochev and M. D. Gunzburger, (1998) Finite element methods of leastsquares type, SIAM Review 40 (1998), 789.

[4] P. Grisvard, Elliptic problems in nonsmooth domains, Pitmann Press, Boston, 1985.

[5] V. Pereyra and G. Scherer, Least squares collocation solution of elliptic problems in general regions, Math Comput Simulation 73 (2006) 226.

[6] P. Žitñan, Vibration analysis of membranes and plates by a discrete least squares technique, J Sound Vib 195 (1996), 595.

[7] M. A. Taylor, B. A. Wingate and R. E. Vincent, An algorithm for computing Fekete points in the triangle, SIAM J Numer Anal 38 (2000), 1707.

[8] L. Bos, S. De Marchi, M. Vianello and Y. Xu, Bivariate Lagrange interpolation at the Padua points: The ideal theory approach, Numer. Math. 108 (2007), 43.

[9] L. Bos, M. Caliari, S. De Marchi, M. Vianello, A numerical study of the Xu polynomial interpolation formula in two variables, Computing 76 (2006), 311.

[10] M. Caliari, S. De Marchi, M. Vianello, Bivariate polynomial interpolation on the square at new nodal sets, Appl Maths Comput 165 (2005), 261.

[11] L. Bos, M. A. Taylor and B. A. Wingate, Tensor product Gauss-Lobatto points are Fekete points for the cube, Math Comput 70 (2001), 1543.

[12] Q. Chen and I. Babuška, Approximate optimal points for polynomial interpolation of real functions in an interval and in a triangle, Comput Methods Appl Mech Engng 128 (1995), 405.

[13] J. S. Hesthaven, From electrostatics to almost optimal points for polynomial interpolation in a simplex, SIAM J Numer Anal 35 (1998), 655.

[14] W. Heinrichs, Improved Lebegue constants on the triangle, J Comput Phys 207 (2005), 625.

[15] M. G. Blyth and C. Pozrikidis, A Lobatto interpolation grid over the triangle, IMA J Appl Math 71 (2006), 153. 
[16] T. Warburton, An explicit construction of interpolation nodes on the simplex, J Eng Math 56 (2006), 247.

[17] Y. Xu, "Lecture notes on orthogonal polynomials of several variables," W. zu Castell, F. Filbir and B. Forster, editors, Advances in the theory of special functions and orthogonal polynomials, Nova Science Publisher, New York, 2004, pp. 135.

[18] E. Bendito, A. Carmona, A. M. Encinas and J. M. Gesto, Estimation of Fekete points, J Comput Phys 225 (2007), 2354.

[19] N. Mai-Duy and T. Tran-Cong, A Cartesian-grid collocation method based on radial-basis-function networks for solving PDEs in irregular domains, Numer Method Partial Differential Equations 23 (2007), 1192.

[20] W. Sun, J. Wu and X. Zhang, Nonconforming spline collocation methods in irregular domains, Numer Method Partial Differential Equations 23 (2007), 1509

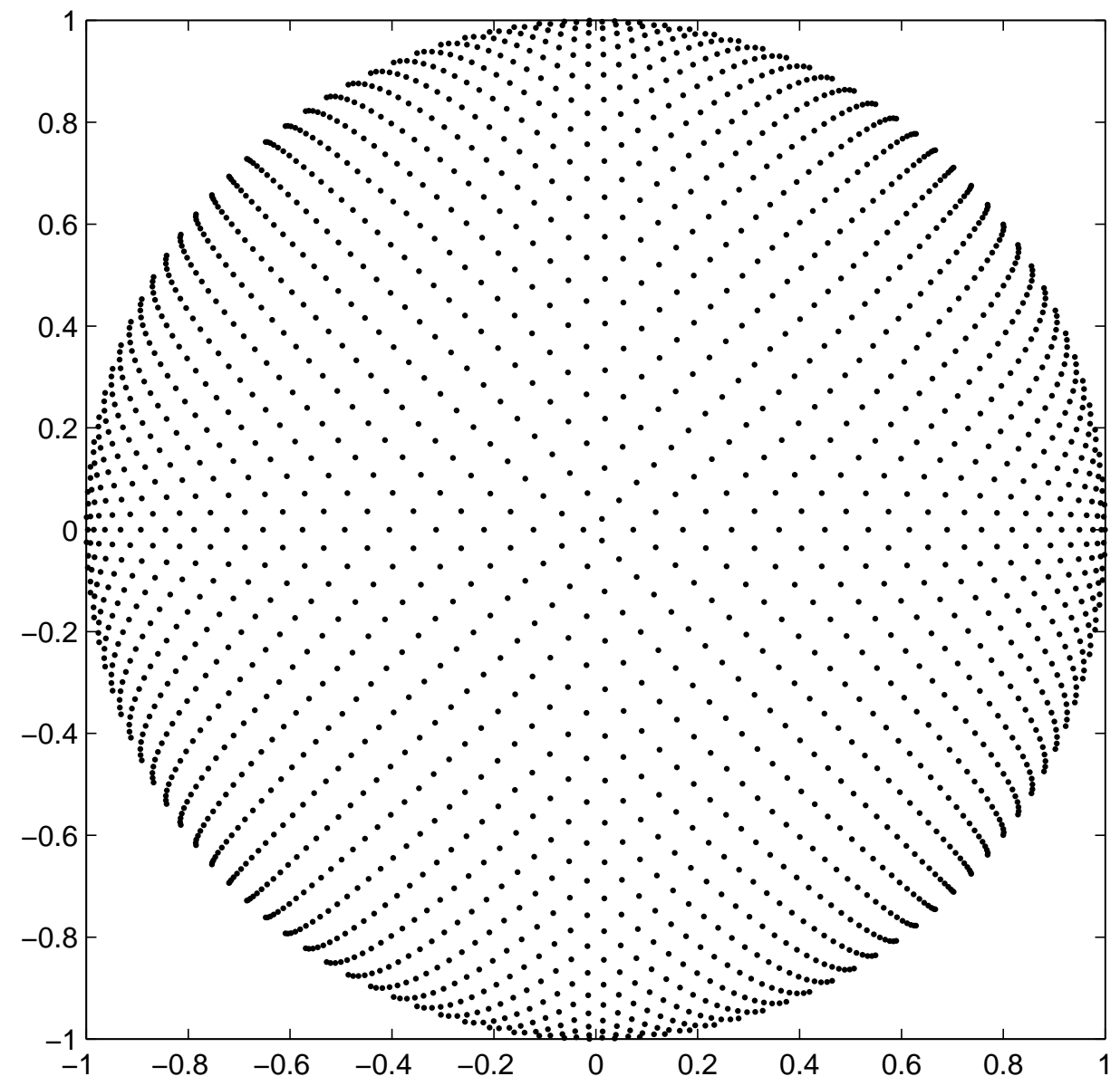

\section{Figure 1.}

2080 FCD-points determined by the parameters $m_{1}=3, m_{2}=4, m_{3}=32$. 


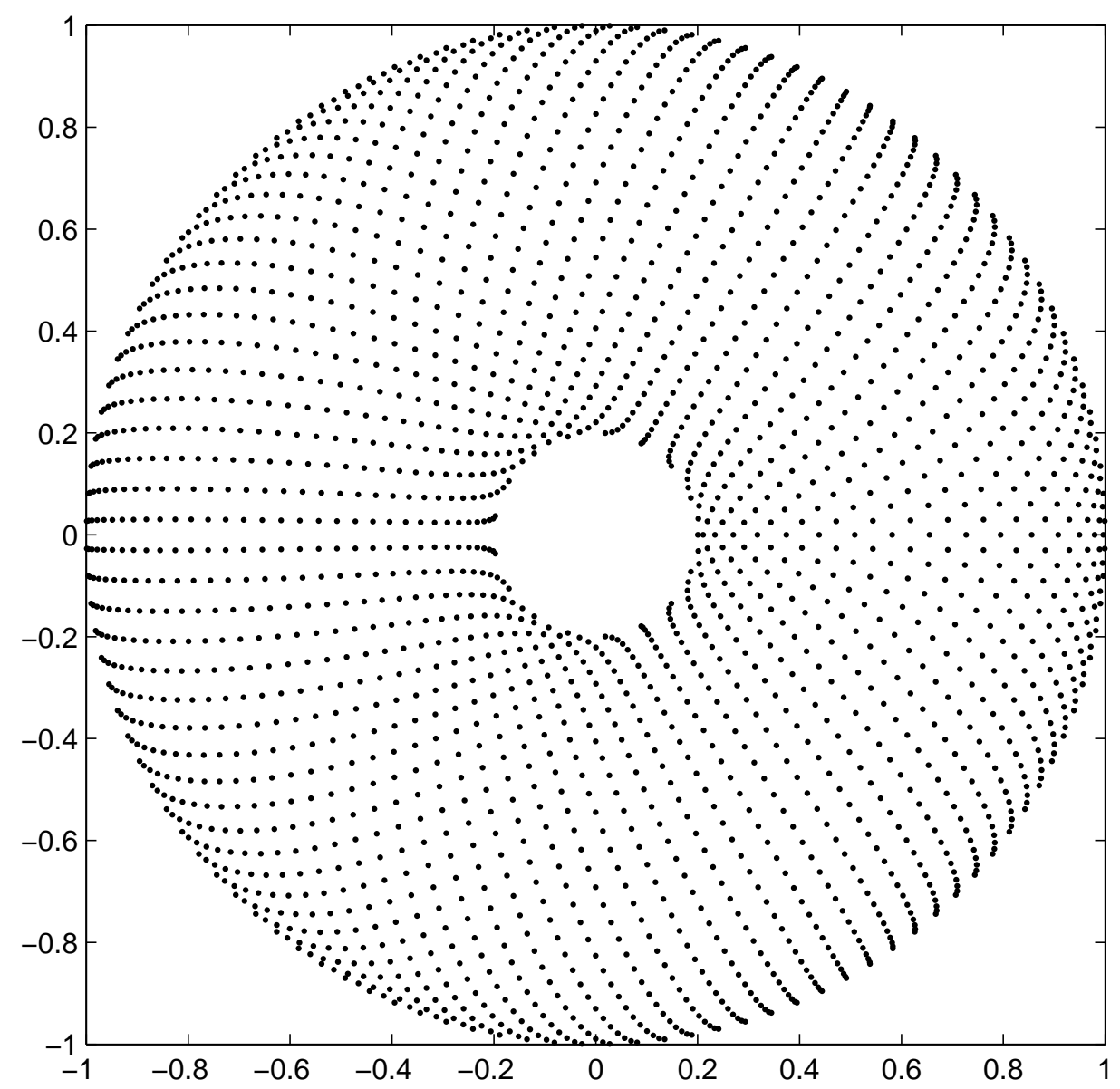

Figure 2.

2261 FCA-points determined by the parameters $m_{1}=17, m_{2}=3, m_{3}=17$. 


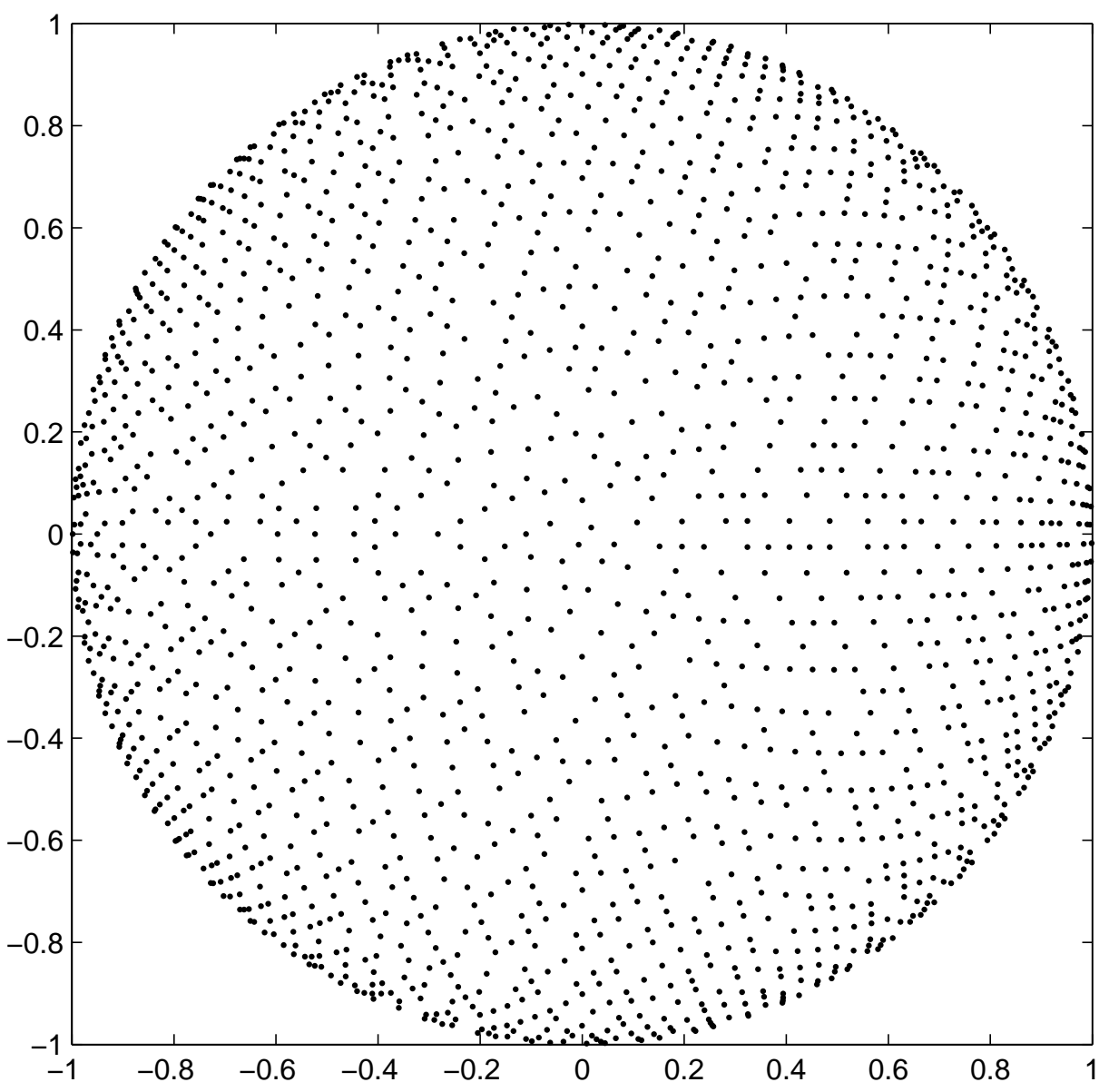

Figure 3.

1800 approximate Fekete points of Sommariva and Vianello selected from 3150 Chebyshev disc points. 
Table 1: Optimized spectral condition numbers $\log _{10}\left(\operatorname{cond}\left(A_{\alpha}\right)\right)$ for the Poisson and biharmonic problems defined on the unit disc using $m$ FCD-points with $n$ basis functions (4) and (8), respectively.

\begin{tabular}{|c|c|c|c|c|c|c|c|c|}
\hline \multirow[b]{2}{*}{$n$} & \multirow[b]{2}{*}{$m_{1}$} & \multirow[b]{2}{*}{$m_{2}$} & \multirow[b]{2}{*}{$m_{3}$} & \multirow[b]{2}{*}{$m$} & \multicolumn{2}{|c|}{ Poisson problem } & \multicolumn{2}{|c|}{ biharmonic problem } \\
\hline & & & & & $\alpha$ & $\log _{10}\left(\operatorname{cond}\left(A_{\alpha}\right)\right)$ & $\alpha$ & $\log _{10}\left(\operatorname{cond}\left(A_{\alpha}\right)\right)$ \\
\hline$\overline{100}$ & 3 & 4 & 7 & 105 & 0.44 & 2.4 & 1.15 & 4.8 \\
\hline 200 & 3 & 4 & 11 & 253 & 0.39 & 2.8 & 1.15 & 5.6 \\
\hline 400 & 3 & 4 & 15 & 465 & 0.27 & 3.3 & 0.93 & 6.7 \\
\hline 800 & 3 & 4 & 22 & 990 & 0.14 & 3.9 & 0.72 & 7.8 \\
\hline 1600 & 3 & 4 & 32 & 2080 & 0.12 & 4.4 & 0.58 & 8.9 \\
\hline
\end{tabular}

Table 2: Error bounds (6) of the Poisson problem defined on the unit disc using $n$ basis functions (4) and $m$ FCD-points reported in Table 1.

\begin{tabular}{lccccc}
\hline \hline$n / m$ & $100 / 105$ & $200 / 253$ & $400 / 465$ & $800 / 990$ & $1600 / 2080$ \\
\hline$\gamma=1$ & -7.9 & -13.5 & -14.1 & -14.1 & -14.2 \\
$\gamma=20$ & -0.1 & -1.0 & -2.2 & -5.8 & -11.8 \\
$\gamma=30$ & 0.6 & -0.1 & -0.4 & -2.9 & -7.0 \\
\hline
\end{tabular}

Table 3: Error bounds (6) of the biharmonic problem defined on the unit disc using $n$ basis functions (8) and $m$ FCD-points reported in Table 1.

\begin{tabular}{lccccc}
\hline \hline$n / m$ & $100 / 105$ & $200 / 253$ & $400 / 465$ & $800 / 990$ & $1600 / 2080$ \\
\hline$\gamma=1$ & -6.4 & -12.6 & -14.2 & -14.1 & -13.5 \\
$\gamma=20$ & 0.7 & -0.3 & -2.1 & -6.1 & -12.3 \\
$\gamma=30$ & 1.6 & 0.7 & -0.2 & -2.8 & -7.9 \\
\hline
\end{tabular}

Table 4: Optimized spectral condition numbers $\log _{10}\left(\operatorname{cond}\left(A_{\alpha}\right)\right)$ for the Poisson and biharmonic problems defined on the unit concentric annulus using $m$ FCA-points and $n$ basis functions (9) and (11), respectively.

\begin{tabular}{|c|c|c|c|c|c|c|c|c|}
\hline \multirow[b]{2}{*}{$n$} & \multirow[b]{2}{*}{$m_{1}$} & \multirow[b]{2}{*}{$m_{2}$} & \multirow[b]{2}{*}{$m_{3}$} & \multirow[b]{2}{*}{$m$} & \multicolumn{2}{|c|}{ Poisson problem } & \multicolumn{2}{|c|}{ biharmonic problem } \\
\hline & & & & & $\alpha$ & $\log _{10}\left(\operatorname{cond}\left(A_{\alpha}\right)\right)$ & $\alpha$ & $\log _{10}\left(\operatorname{cond}\left(A_{\alpha}\right)\right)$ \\
\hline 100 & 3 & 2 & 6 & 168 & 1. & 2.9 & 2. & 4.6 \\
\hline 200 & 7 & 2 & 8 & 352 & 0.73 & 3.5 & 1.67 & 6.1 \\
\hline 400 & 12 & 3 & 8 & 552 & 0.42 & 4.3 & 1.52 & 8.3 \\
\hline 800 & 30 & 2 & 12 & 1272 & 0.37 & 5.5 & 1.37 & 9.4 \\
\hline 1600 & 17 & 3 & 17 & 2261 & 0.35 & 8.3 & 1. & 11.0 \\
\hline
\end{tabular}

Table 5: Error bounds (6) of the Poisson problem defined on the unit concentric annulus using $n$ basis functions (9) and $m$ FCA-points reported in Table 4.

\begin{tabular}{lccccc}
\hline \hline$n / m$ & $100 / 168$ & $200 / 352$ & $400 / 552$ & $800 / 1272$ & $1600 / 2261$ \\
\hline$\gamma=1$ & -7.1 & -13.1 & -13.9 & -13.3 & -13.5 \\
$\gamma=20$ & 0.8 & -0.2 & -1.9 & -5.5 & -10.9 \\
$\gamma=30$ & 1.2 & 0.7 & -0.1 & -2.4 & -6.3 \\
\hline
\end{tabular}


Table 6: Error bounds (6) of the biharmonic problem defined on the unit concentric annulus using $n$ basis functions (11) and $m$ FCA-points reported in Table 4.

\begin{tabular}{lccccc}
\hline \hline$n / m$ & $100 / 168$ & $200 / 352$ & $400 / 552$ & $800 / 1272$ & $1600 / 2261$ \\
\hline$\gamma=1$ & -7.4 & -12.8 & -12.1 & -11.7 & -11.3 \\
$\gamma=20$ & 0.9 & -0.2 & -2.0 & -5.6 & -9.6 \\
$\gamma=30$ & 1.8 & 1.2 & -0.1 & -2.5 & -6.5 \\
\hline
\end{tabular}

Table 7: Spectral condition numbers $\log _{10}\left(\operatorname{cond}\left(A_{\alpha}\right)\right)$ of the non-optimized $(\alpha=0)$ and optimized $(\alpha=1.8)$ coefficient matrices $A_{\alpha}$ for the Poisson problem defined on the unit disc using $n$ basis functions (12) and $m$ approximate Fekete points.

\begin{tabular}{rcccrcccc}
\hline$n$ & $m_{1}$ & $m_{2}$ & $m_{3}$ & \multicolumn{1}{c}{$M$} & \multicolumn{1}{c}{$m$} & $n b p$ & $\log _{10}\left(\operatorname{cond}\left(A_{0}\right)\right)$ & $\log _{10}\left(\operatorname{cond}\left(A_{1.8}\right)\right)$ \\
\hline 100 & 3 & 3 & 23 & 828 & 120 & 25 & 5.1 & 2.5 \\
200 & 3 & 3 & 23 & 828 & 240 & 40 & 6.2 & 3.1 \\
400 & 3 & 3 & 33 & 1683 & 440 & 60 & 7.2 & 3.6 \\
800 & 5 & 5 & 35 & 3150 & 880 & 80 & 7.9 & 4.1 \\
1600 & 5 & 5 & 35 & 3150 & 1800 & 120 & 8.5 & 4.5 \\
\hline
\end{tabular}

Table 8: Error bounds (6) of the Poisson problem defined on the unit disc using $n$ basis functions (12) and $m$ approximate Fekete points reported in Table 7.

\begin{tabular}{lccccc}
\hline \hline$n / m$ & $100 / 120$ & $200 / 240$ & $400 / 440$ & $800 / 880$ & $1600 / 1800$ \\
\hline$\gamma=1$ & -5.7 & -9.3 & -13.7 & -14.5 & -14.0 \\
$\gamma=20$ & 0.3 & -0.6 & -2.1 & -5.1 & -10.6 \\
$\gamma=30$ & 0.9 & 0.4 & -0.3 & -2.4 & -6.3 \\
\hline
\end{tabular}

Table 9: Spectral condition numbers $\log _{10}\left(\operatorname{cond}\left(A_{\alpha}\right)\right)$ of the non-optimized $(\alpha=0)$ and optimized $(\alpha=3.8)$ coefficient matrices $A_{\alpha}$ for the biharmonic problem defined on the unit disc using $n$ basis functions (12) and $m$ approximate Fekete points.

\begin{tabular}{rcccccccc}
\hline$n$ & $m_{1}$ & $m_{2}$ & $m_{3}$ & $M$ & \multicolumn{1}{c}{$m$} & $n b p$ & $\log _{10}\left(\operatorname{cond}\left(A_{0}\right)\right)$ & $\log _{10}\left(\operatorname{cond}\left(A_{3.8}\right)\right)$ \\
\hline 100 & 3 & 3 & 33 & 1633 & 120 & 70 & 8.5 & 4.6 \\
200 & 3 & 3 & 33 & 1683 & 240 & 70 & 11.2 & 6.1 \\
400 & 4 & 4 & 44 & 3960 & 440 & 100 & 12.7 & 7.0 \\
800 & 4 & 4 & 44 & 3960 & 880 & 100 & 14.9 & 8.1 \\
1600 & 4 & 4 & 44 & 3960 & 1800 & 150 & 17.6 & 9.2 \\
\hline
\end{tabular}

Table 10: Error bounds (6) of the biharmonic problem defined on the unit disc using $n$ basis functions (12) and $m$ approximate Fekete points reported in Table 9.

\begin{tabular}{lccccc}
\hline \hline$n / m$ & $100 / 120$ & $200 / 240$ & $400 / 440$ & $800 / 880$ & $1600 / 1800$ \\
\hline$\gamma=1$ & -3.2 & -6.4 & -10.9 & -12.1 & -12.3 \\
$\gamma=20$ & 1.0 & 0.3 & -1.6 & -4.4 & -10.3 \\
$\gamma=30$ & 1.6 & 1.1 & 0.2 & -1.6 & -5.9 \\
\hline
\end{tabular}

